\title{
EQUIPMENTS AND SUPPORT SYSTEMS FOR INTERVENTION IN EMERGENCY SITUATIONS - THE CONCEPTUAL SCHEME
}

\author{
ADRIAN SĂLIŞTEAN, DOINA TOMA, CLAUDIA NICULESCU, SABINA OLARU \\ The National Research Development Institute for Textiles and Leather, 16, Lucretiu Patrascanu, \\ 030508 Bucharest, Romania; e-mail: certex@certex.ro,webpage: http://www.certex.ro
}

\begin{abstract}
The aim of the project is strengthening the operational response capacity of the National Emergency Situation Management System structures. The objective is to develop and implement the integrated support equipment and systems in an architecture that meets the technical and especially operational requirements specific to emergency response actions. The concept is an integrated system with two components: protective equipment and support system for intervention actions. The originality is the support system as a complex UAS that consists of one or more ultralight multifunctional sail air vectors with a configuration that can be adapted to the nature of the intervention: monitoring, observation and logistics, air transport, detection of intervention staff using the PPE that is equipped with a special radio ID transponder. PPE must respond to increasingly evolved and varied threats that act on users. PPE are designed to remain effective and functional across a wide range of tactical and operating environments: from arctic temperatures in the desert, high and low humidity, in urban and rural environments, forested, marine, underground and almost in all imaginable locations. The novelty of PPE is the modular multilayer structure made of yarns / fibers with integrated protection properties (flame resistance and / or cutting resistance/ water, chemical and body fluid rejection/ antimicrobial protection etc.).
\end{abstract}

Keywords: Unmanned Aerial System (UAS), Personal protective equipment (PPE), Emergency response

\section{INTRODUCTION}

Emergency response is a series of organized and coordinated precaution and actions during the time between the detection of possible event and stabilizing the situation. An emergency response decision support system needs to assist decision makers to evaluate emergency plans and select an appropriate plan of action during an emergency by supporting heterogeneous emergency response data sources and providing decision makers with access to appropriate emergency rescue knowledge. It also needs to provide differentiated services to meet particular requirements.

Decision making in emergency response is an extremely time-sensitive and challenging task that requires immediate and effective response from decision makers who are surrounded by a variety of uncertain information and are under huge pressure from the need to coordinate action. An emergency response decision support system needs to assist decision makers to evaluate emergency plans and select an appropriate plan of action during an emergency by supporting heterogeneous emergency response data sources and providing decision makers with access to appropriate emergency rescue knowledge. It also needs to provide differentiated services to meet particular requirements.

There are four main emergency response functions - emergency assessment, risk operations, population protection and incident management. The four functions provide a framework for organizing response activities to a wide variety of emergencies, natural hazards, technological accidents, terrorist attacks, and sabotage.

- Emergency assessment: The emergency response activities in the response phase relate to the understanding of the behavior of the hazard-generating factors but also of the risk to human life and material damage. 
- Risk operations: Risk operations aim at mitigating emergency situations but are only implemented when needed. Their applicability varies greatly from one hazard to another.

- Protection of the population: The information collected during the emergency assessment is the basis for the choice of population protection actions.

- Incident management: Incident management involves the development of an incident management policy, a set of consistent, repeatable, measurable processes and procedures and the use of appropriate administrative, managerial, technical or legal means to detect analyze and respond to incidents serious.

Regardless of the emergency structure that acts against the timer, time is the greatest enemy, and the scoring scale is the response time, which must be very low. With the latest Unmanned Aerial Vehicles (UAV) technology, the risks that influence response time can be reduced.

Also unmanned aerial devices allow rapid surveillance of dangerous situations, risk analysis and assessment can provide information to control centers, being an eye in the sky.

Starting from the premise that the way of manifestation of the emergency situations and their management system in the future will be very different from the past context, we have proposed to develop an integrated support equipment and systems that respond to the challenges and needs of these field that need to be addressed in an organized and integrated manner.

\section{EXPERIMENTAL PART}

\section{Operational and Performance Requirements for an Integrated System}

The Emergency Assessment is the first phase with the following operating requirements:

- A1: Collecting information from where events occurred: fires, explosions, industrial accidents, floods, etc.;

- A2: Detection of the NBC contamination level of an area;

- A3: Patrolling of some areas (border, communication routes, infrastructure electrical networks, transport pipelines etc.) for the purpose of preventive detection of emergency situations.

On the basis of the collected data, it is possible to move to the second phase of efficient incidents management through:

- B1: Persistent surveillance of the area where events occur that have a continuous spatial and temporal evolution (fires, floods, natural disasters, industrial accidents, etc.);

- B2: Appropriate equipment for intervention staff with PPE tailored specifically to the event produced;

- B3: Locating and tracking in real time intervention teams;

- B4: Search for missing persons in natural environments covered with dense vegetation;

- B5: Temporary provision of radio communication coverage of mobile radio communications networks in isolated / hard-to-reach areas or where terrestrial networks are unavailable / degraded;

- B6: Small-scale logistics transport in remote areas. 


\section{Component and Capabilities of the Integrated System Support}

The conceptual block scheme of the integrated support system for emergency interventions is presented in Figure 1.

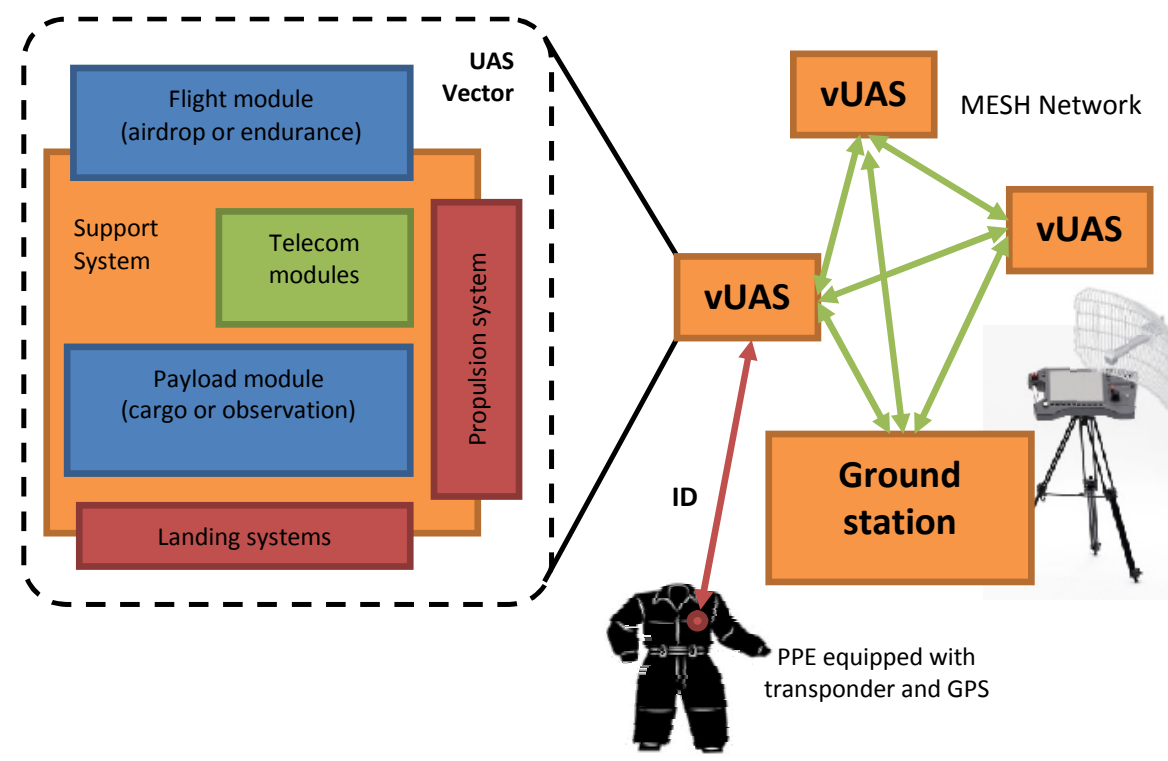

Figure 1. The conceptual block scheme of the integrated support system for emergency interventions

\section{Personal Protective Equipment}

Protective equipment must remain effective and functional in a wide range of tactical and operating environments: from arctic to desert temperatures, high and low humidity, in urban and rural environments, forested, marine, underground and almost all locations imaginable.

In the development of protective equipment for intervention, innovative solutions have been applied, resulting in a modular protective clothing system, customized for various emergency response environments.

The modular system consists of:

- The service uniform (base layer) that provides risk protection with the highest probability of occurrence in case of an intervention action;

- Modular outer layers specific to the type of intervention mission that is physically modular but performs unitarily and fully functional within an IPE system, depending on the specificity of the intervention mission. 


\section{Support System in Intervention Actions}

The innovative solution applied to the development of support systems for emergency intervention consists of a UAS support system with adaptable configuration depending on the nature of the intervention:

- An endurance flight module made of high glide paraglider wing;

- A para-drop unit, in particular a ram-air parachute attached to the automated control unit.

- Upgrading the detection capability as required: dedicated sensors for NBC; attaching a transponder to detect and locate intervention staff using protective clothing.

\section{Component Elements of the Support System}

1) UAS vectors (vUAS)

UAV, flexible wing type paraglider, with dual-electrically / thermally propelled and interchangeable load:

- C1: Video stabilized support: HD day-night and / or FLIR (Forward looking infrared) camera and / or LiDAR (Light Detection and Ranging) sensor;

- C2: NBC sensor suite including: gas detector and volatile organic compounds; ionizing radiation detector and aerosol sample collector;

- C3: Detection and location unit for multiband and telecommunication relay in GSM band;

- C4: Cargo unit.

2) The PPE transponder

The transponder is a miniature device within the PPE, with the role of receiving the GPS signal and then retransmits it with a unique identification number.

3) Command and Control Station (SCC)

This station is used for controlling and monitoring the air vectors and transmitting the data in real time to the command point of the intervention and consists of: antennas (a fixed antenna and a tracking antenna); data transceiver; microcomputer; HID (Human Interface Devices); rechargeable battery and generator. Transmission of these data is encrypted by high-speed terrestrial data transmissions in the $5 \mathrm{GHz}$ or $4 \mathrm{G}$ band if there is no access to a terrestrial telecommunication hub.

\section{RESULTS AND DISCUSSION}

From the analysis of the situations faced by the Flexible Wing (Knache, 1992), it has emerged that this must be a hybrid ram-air paraglider. This type of wing harmoniously combines the performance of a classic dual skin wings with that of a single skin wings, figure 2. The paraglider wing profile is the main determinant of flight performance (Poynter, 1984).

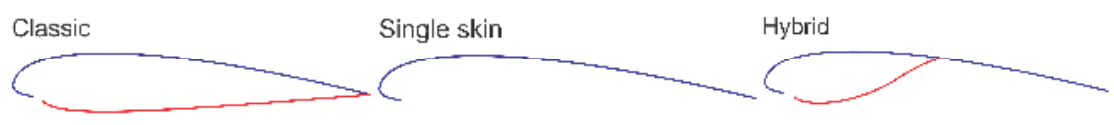

Figure 2. Types of flexible wing profiles 
A flat wing was designed with the attack board cut off to create the air circulation inside the canopy volume. We made the following estimates:

- the load factor has been preserved the same as for the flat wing;

- the drag coefficient CD0 changes substantially, it can vary between $(0,5 \div 1,0)$

CD0 for the flat wing.

Projected span of flexible wing: $6.5 \mathrm{~m}$

\section{CONCLUSION}

1. The modular configuration of UAS support system and load variants of the UAS support system are:

- C1: video suite: permanently mounted (for observation, monitoring);

- C2: NBC sensor set: if necessary (for investigation area, NBC hazard detection);

- C3: sensor detection and localization sensor set: if necessary (for locating missing persons, fire detection and wind direction detection);

- C4: cargo unit: if necessary (or emergency transport, medicines and supplies in remote areas, small cargo, up to $10 \mathrm{~kg}$ ).

2. The flexible ram-air wing will be a hybrid ram-air glider with 6.5 meter span.

\section{Acknowledgements}

This work was done on NUCLEU program, TEX-PEL-2020, implemented with the Ministry of Research and Innovation support, project no. 16N/18 230301.

\section{REFERENCES}

Green, L.V. and Kolesar, P.J. (2004), "Improving emergency responsiveness with management science", Manage Sci, 50(8), 1001-1014, https://doi.org/10.1287/mnsc.1040.0253.

Knache, T.W. (1992), Parachute Recovery Systems - Design Manual, Para Publishing, Santa Barbara, California.

Poynter, D. (1984), The Parachute Manual - A Technical Treatise on Aerodynamic Decelerators, Vol.2, Santa Barbara, California. 